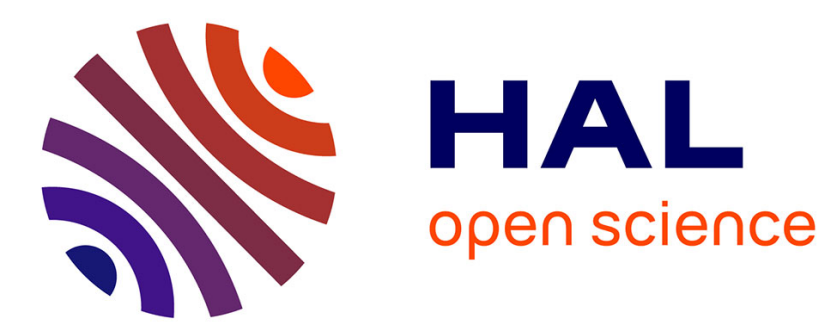

\title{
Influence of bubble size and thermal dissipation on compressive wave attenuation in liquid foams
}

\author{
Martin Monloubou, Arnaud Saint-Jalmes, Benjamin Dollet, Isabelle Cantat
}

\section{To cite this version:}

Martin Monloubou, Arnaud Saint-Jalmes, Benjamin Dollet, Isabelle Cantat. Influence of bubble size and thermal dissipation on compressive wave attenuation in liquid foams. EPL - Europhysics Letters, 2015, 112 (3), pp.34001. 10.1209/0295-5075/112/34001 . hal-01230039

\section{HAL Id: hal-01230039 \\ https://hal.science/hal-01230039}

Submitted on 17 Nov 2015

HAL is a multi-disciplinary open access archive for the deposit and dissemination of scientific research documents, whether they are published or not. The documents may come from teaching and research institutions in France or abroad, or from public or private research centers.
L'archive ouverte pluridisciplinaire HAL, est destinée au dépôt et à la diffusion de documents scientifiques de niveau recherche, publiés ou non, émanant des établissements d'enseignement et de recherche français ou étrangers, des laboratoires publics ou privés. 


\title{
Influence of bubble size and thermal dissipation on compressive wave attenuation in liquid foams
}

\author{
M. Monloubou ${ }^{1}$, A. Saint-Jalmes ${ }^{1}$, B. Dollet ${ }^{1}$ and I. Cantat ${ }^{1}$ \\ 1 Institut de Physique de Rennes, UMR 6251 CNRS / Université de Rennes 1, Rennes, France.
}

PACS 47.57.Bc - Foams; Complex fluids

PACS 43.25.Ed - Acoustical absorption; Nonlinear acoustics

\begin{abstract}
Acoustic or blast wave absorption by liquid foams is especially efficient and bubble size or liquid fraction optimization is an important challenge in this context. A resonant behavior of foams has recently been observed, but the main local dissipative process is still unknown. In this paper, we evidence the thermal origin of the dissipation, with an optimal bubble size close to the thermal boundary layer thickness. Using a shock tube, we produce typical pressure variation at time scales of the order of the millisecond, which propagates in the foam in linear and slightly non-linear regimes.
\end{abstract}

Liquid foams have a surprisingly high effective viscosity, which can be several orders of magnitude larger than the foaming solution viscosity [1]. Energy dissipation in foams may have very different local origins and their identification, as a function of the characteristic time scales and of the foam properties, is a long lasting fundamental and applied challenge [2]. For simple shear, dissipation has been shown to be directly coupled to the foam coarsening at very low frequency $\left(10^{-2} \mathrm{~Hz}\right)[3,4]$ and to arise from viscous dissipation between bubbles for frequencies around the $\mathrm{Hz}[5]$. At higher frequencies, the foam loss modulus varies as the square root of the frequency and the local origin of this dissipation is still a matter of debate [6-9]. For isolated films vibrating at $1 \mathrm{kHz}$, the viscous dissipation in air is dominant [10].

Compressive waves in foams have been studied in the context of acoustic and blast waves. The need to optimize the blast wave absorption, in terms of liquid fraction, bubble size or chemical formulation, stimulated experimental studies at the film scale [11] and at the foam scale, both on field, using real detonations $[12,13]$, and in the lab, using shock tubes $[14,15]$. Most theoretical approaches on blast wave absorption disregard the foam structure, and focus only on the role of the liquid fraction [16]. In the acoustic pressure range however, several studies clearly established the important role of the bubble size on the sound propagation, both theoretically and experimentally [17-22].

Compression waves modify the gas temperature and thus induce thermal effects. Thermal dissipation is exacerbated in the foam, as its structure makes especially efficient heat transfer between the liquid and gas phases. It has been computed for a bubbly liquid, in the limit of small gas fraction, evidencing two limiting cases, for bubbles of radius $R$ much smaller or much larger than the thermal length $\ell_{T}=\sqrt{\chi \tau}$, with $\tau$ the excitation characteristic time and $\chi$ the heat diffusivity. The attenuation coefficient is predicted to scale as $R^{2} / \tau^{2}$ for small bubbles (isothermal regime), and as $1 /(R \sqrt{\tau})$ for large ones (isentropic regime) [23,24]. A good agreement has been found with the first scaling for acoustic waves of frequencies in the range $40-80 \mathrm{kHz}$ propagating in commercial shaving foams [18].

This paper focuses on the attenuation of a compressive wave propagating in a liquid foam, with an overpressure duration $\tau$ of the order of one millisecond. We work on the moderate overpressure regime, below the critical pressure at which the foam is fully destroyed, and above the linear regime. Recording the pressure evolution in a foam sample, we determine the wave amplitude attenuation. We establish the scaling laws for the thermal attenuation in the context of dry foams and recover the two scaling laws proposed for bubbly liquids [24]. Experimentally, the attenuation varies as $R^{2}$ for small bubbles as in [18], but we evidence that it departs from this law for larger bubbles. We thus identify the cross-over between the isothermal and isentropic regimes, for $R \approx 5 \ell_{T}$, that coincides with an optimal energy absorption, distinct from the optimal energy absorption induced by the resonance $[19,21,25]$.

The foam is produced by turbulent mixing of nitrogen and foaming solution at a flow rate of the order of 100 


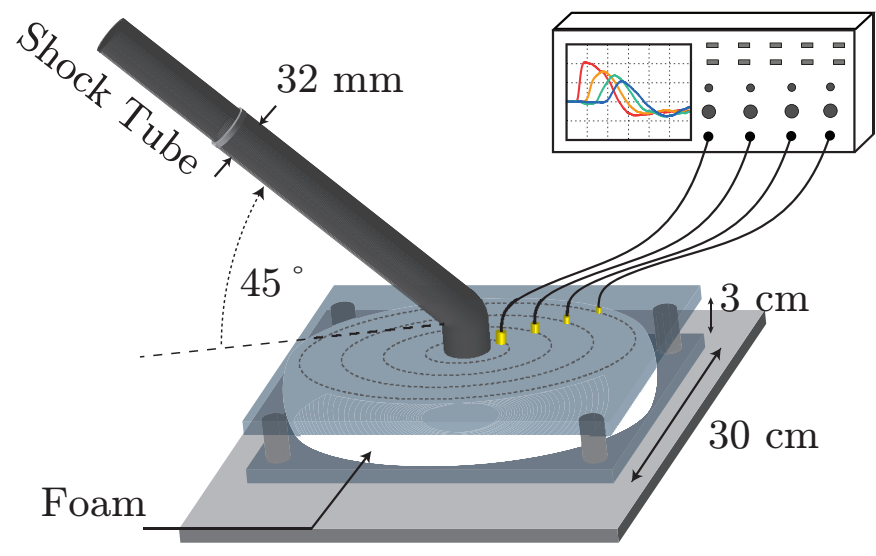

Fig. 1: Sketch of the experimental set-up.

$\mathrm{mL} / \mathrm{s}[26]$. The solution is composed of SDS (sodium dodecyl sulfate) at a concentration $10 \mathrm{~g} / \mathrm{L}$ in pure water. The foam is then injected in a cell made of two transparent square plates, through a hole in the middle of the top plate. The plates are $30 \mathrm{~cm}$ wide and the gap between them is $H=3 \mathrm{~cm}$ (see Fig. 1). The liquid fraction of the foam is controlled by the nitrogen flux and is measured a posteriori. Its value is $\phi_{l}=(4.8 \pm 0.3) \%$ for all the results shown in the paper. As the foam evolves by coarsening, the average bubble size increases with time and bigger bubbles are obtained after some delay. During this delay, the cell is flipped upside down every 30 seconds to ensure a homogeneous liquid fraction in the cell. With this method, the accessible bubble radius is $100-300 \mu \mathrm{m}$. Larger bubble radii, up to $500 \mu \mathrm{m}$, have been obtained by blowing nitrogen through four porous frits immersed in the foaming solution, below the cell, held vertically.

Just after the shock, a few hundreds of bubbles are extracted from the cell and spread on a thin layer of foaming solution, forming a monolayer of spherical bubbles. Their average radius $R$, determined by image processing, is used to quantify the bubble size in the foam [25]. The diameter distribution is roughly log-normal, with a normalized standard deviation of the order of 0.4.

A pressure wave is generated with a home-made shock tube inspired by [27], composed of a high pressure chamber separated from a low pressure one by a diaphragm. Nitrogen is injected into the high pressure chamber until the rupture of the diaphragm, which generates a shock wave (i. e. an overpressure step) that propagates in the tube. When reaching the exit of the tube, which is connected to the middle of the cell (see Fig. 1), the shock wave turns into a blast wave that propagates in the foam. The wave amplitude is varied by changing the diaphragm. We used aluminium foil (1 to 4 layers) and, for the weakest shocks, one parafilm layer. Four pressure sensors $P C B 113$ B28 are installed on the top plate at a distance $r_{i}$ from the centre, with $i=[1-4]$ and $r_{i}=[3.10,5.15,7.20,9.25] \mathrm{cm}$. They are connected to an oscilloscope TDS $2004 \mathrm{~B}$, and record the pressure $p_{i}(t)$ with a sampling time of $4 \mu \mathrm{s}$.
Fig. 2 shows typical pressure signals as a function of time, at different distances from the cell center. At sensor 1 , a rapid pressure increase is followed by a slower pressure decrease and a small underpressure. This kind of profile is typical of a blast wave [12]. The duration of the overpressure is of the order of $2 \mathrm{~ms}$ and finishes before the first wave reflections come back from the external foam boundary, in contact with air. The results do not depend on the cell thickness $H$ for $H<5 \mathrm{~cm}$ and the wave propagation is cylindrical.

The transmission coefficient from the shock tube to the foam depends on the bubble size and on the shock amplitude, but the rescaled pressure $p_{1}(t) / P_{1}$, with $P_{1}$ the maximal pressure at sensor 1 , is independent on those parameters (see Fig. 2, inset). The initial signal $p_{1}(t)$ is thus fully characterized by the single parameter $P_{1}$ which is our control parameter for the shock amplitude in the whole study.

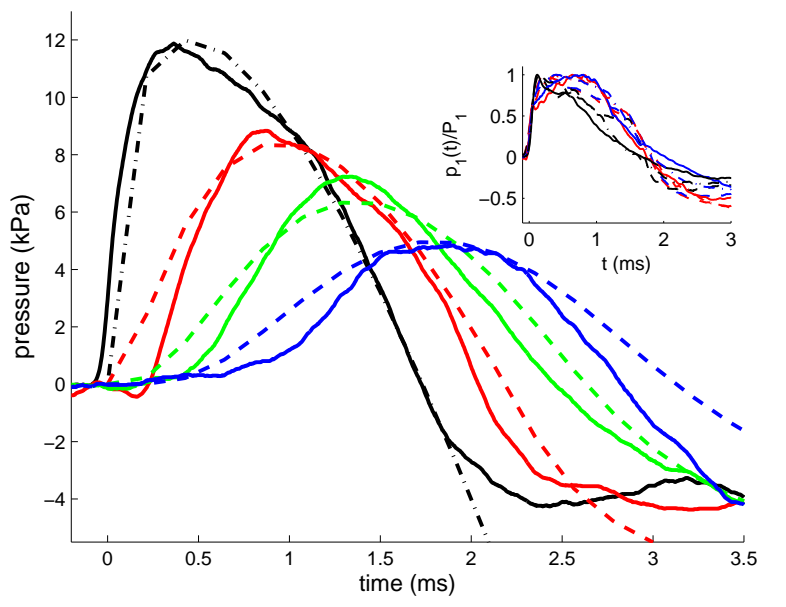

Fig. 2: Pressure at sensors 1 to 4 (solid lines, from left to right) as a function of time for $R=210 \mu \mathrm{m}$. Dash-dotted line: signal used as numerical boundary condition for the simulations. Dashed lines: numerical simulations of the pressure at sensor 2 to 4 , with $\nu=1.1 \mathrm{~m}^{2} / \mathrm{s}$ and $\gamma=1$. Inset: rescaled signals $p_{1}(t) / P_{1}$ at sensor 1 for $R=115$ (black), 210 (blue) and 265 (red) $\mu \mathrm{m}$ for 4 aluminium foils (solid line), 2 foils (dash-dotted) and parafilm (dashed) (color online).

The pressure ratio, $\alpha=P_{4} / P_{1}$, with $P_{4}$ the maximum pressure value measured at sensor 4 , is shown in Fig. 3 as a function of the amplitude, for 6 bubble sizes. $\alpha$ increases with the amplitude and can be fitted by a phenomenological affine law $\alpha^{\text {fit }}\left(R, P_{1}\right)=\alpha^{0}(R)+K P_{1}$, with $K=6.5 \mathrm{~Pa}^{-1}$ the slope leading to the best agreement. The standard deviation is $\sigma(R)=\left\langle\left(\alpha^{e x p}-\alpha^{f i t}\right)^{2}\right\rangle^{1 / 2}$. In the linear regime, the wave attenuation is classically quantified by an attenuation length $\ell_{a}$ defined by the relation $\alpha=\alpha^{r e f} \exp \left[\left(r_{1}-r_{4}\right) / \ell_{a}\right], \alpha^{r e f}$ being the expected attenuation, in the non-dissipative case. In our cylindrical geometry, $\alpha^{r e f}=\left(r_{1} / r_{4}\right)^{1 / 2}=0.58$. The length $\ell_{a}^{e x p}$ was computed for each bubble size using the value of $\alpha$ extrap- 


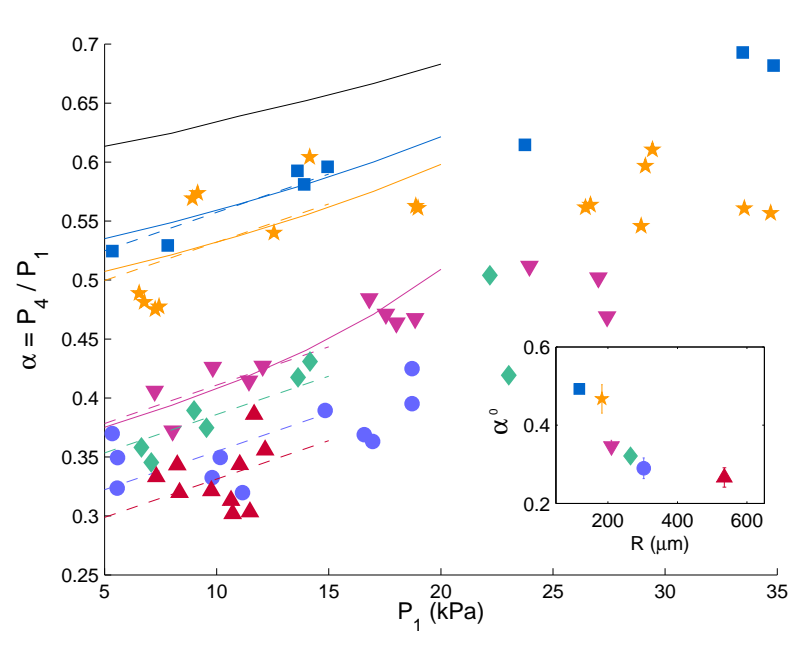

Fig. 3: Pressure ratio in the foam $\alpha=P_{4} / P_{1}$ as a function of the initial wave amplitude $P_{1}$, for different bubble sizes $R$ : $117(\square), 182(\star), 210(\nabla), 265(\diamond), 303(\bullet)$, and $535(\Delta) \mu \mathrm{m}$. The last two points have been obtained with the vertical cell. The liquid fraction is $\phi_{l}=(4.8 \pm 0.3) \%$. The solid lines are numerical results obtained for $\nu=0.001,0.17,0.25$ and 1.5 $\mathrm{m}^{2} / \mathrm{s}$ (from top to bottom) and $\gamma=1$. The dashed line are phenomenological fits $\alpha^{f i t}=\alpha^{0}(R)+6.5 P_{1}$ (in $\mathrm{Pa}$ ). Inset: $\alpha^{0}(R) \pm \sigma(R)$ as a function of the bubble size.

olated at small amplitude, $\alpha^{0}(R) \pm \sigma(R)$, shown in Fig. 3 (inset). The main result is that the pressure attenuation increases with bubble size for bubbles smaller than $300 \mu \mathrm{m}$ (and the attenuation length thus decreases) and saturates for larger bubbles, at constant liquid fraction. The experimental pressure ratio, in the small amplitude limit, remains below the geometrical limit $\alpha^{\text {ref }}$ as expected. However, at larger amplitudes the non-linearities reduce the attenuation, and $\alpha$ may cross this limit.

When the wave reaches a bubble, its pressure increases by typically $\delta p \approx 10^{4} \mathrm{~Pa}$ during $\tau \approx 1 \mathrm{~ms}$. Considering an ideal gas behavior in the bubbles, this may lead to a temperature increase of $\delta T^{s}=\delta P /\left(\rho_{g} c_{p}\right) \approx 10 \mathrm{~K}$ if the process is isentropic, with $\rho_{g}=1.2 \mathrm{~kg} / \mathrm{m}^{3}$ the gas density and $c_{p}=10^{3} \mathrm{~J} \cdot \mathrm{kg}^{-1} \cdot \mathrm{K}^{-1}$ its specific heat at constant pressure. The bubble is surrounded by the liquid phase, that plays the role of a thermostat at temperature $T$. Indeed, in a given volume of foam, the ratio of the thermal capacities of gas and liquid phases equals $\rho_{g} c_{p} /\left(\rho_{s} c_{p, s} \phi_{l}\right)=5 \cdot 10^{-3} \ll 1$, with $\rho_{s}=10^{3} \mathrm{~kg} / \mathrm{m}^{-3}$ the density of the solution and $c_{p, s}=4 \cdot 10^{3} \mathrm{~J} \cdot \mathrm{kg}^{-1} \cdot \mathrm{K}^{-1}$ its specific heat. At a time $\tau$ after the bubble compression, the thickness of the thermal boundary layer is $\ell_{T}=\sqrt{\chi \tau} \approx 10^{-4} \mathrm{~m}$, with $\chi=20 \cdot 10^{-6} \mathrm{~m}^{2} / \mathrm{s}$ the heat diffusivity. This length $\ell_{T}$ is much smaller than the wave length $\lambda=c \tau \approx 5 \cdot 10^{-2} \mathrm{~m}$, with $c \approx 50 \mathrm{~m} / \mathrm{s}$ the sound velocity (see inset of Fig. 4). It is, in contrast, just in the range of the investigated bubble size.

Thermal properties first influence the foam effective compressibility, and thus the wave velocity. A classical prediction for the linear sound velocity $c$ is the Wood velocity. In this model, the foam is replaced by a continuous medium of density $\rho=\phi_{l} \rho_{s}$ related to the local pressure $p^{\text {tot }}$ by a constitutive relation $[28,29]$

$$
p^{\mathrm{tot}}=p_{0}\left[\frac{\left(1-\phi_{l, 0}\right) \rho}{\rho_{0}-\phi_{l, 0} \rho}\right]^{\gamma},
$$

with $p_{0}$ the initial atmospheric pressure, $\rho_{0}$ and $\phi_{0}$ the foam density and its liquid fraction at $p^{\text {tot }}=p_{0}$. The gas can be modeled as a ideal diatomic gas and $\gamma=1.4$ or $\gamma=1$ for the isentropic and isothermal cases, respectively. The Wood velocity is

$$
c_{\mathrm{W}}^{2}=\left(\frac{\partial p^{\mathrm{tot}}}{\partial \rho}\right)_{\rho=\rho_{0}}=\frac{\gamma p_{0}}{\left(1-\phi_{l, 0}\right) \phi_{l, 0} \rho_{s}} .
$$

For $\phi_{l}=0.048, c_{\mathrm{W}}^{t}=47 \mathrm{~m} / \mathrm{s}$ for $\gamma=1$, and $c_{\mathrm{W}}^{s}=56 \mathrm{~m} / \mathrm{s}$ for $\gamma=1.4$. The small amplitude front velocity, measured optically for a better precision (see Appendix A), is better fitted with the isothermal Wood velocity for small bubbles and by the isentropic velocity for large bubbles (see inset of Fig. 4). Note that the front velocity we measured may be sligthly larger than the average velocity of the whole signal, because of the signal spreading. It thus should be considered as an upper boundary for the sound velocity.

More importantly, thermal gradients also lead to energy dissipation and significantly contribute to the wave attenuation. The isentropic and isothermal assumptions are valid if the bubble size is respectively much larger and much smaller than $\ell_{T}$ [24]. We thus derive the scaling laws of the thermal dissipation in the linear regime for small bubbles (isothermal model) and for large bubbles (isentropic model). We then extrapolate these two limiting cases to cover our whole bubble size range.

The mechanical wave carries the energy per bubble $E_{0} \sim \rho_{0} u^{2} R^{3} \sim \rho_{0}\left[\delta p /\left(\rho_{0} c\right)\right]^{2} R^{3}$. This energy decreases with time as [32] $\dot{E} \sim \frac{\kappa}{T} \nabla T^{2} \Omega_{d i s}$ with $\kappa=$ $2 \cdot 10^{-2} \mathrm{~W} \cdot \mathrm{m}^{-1} \cdot \mathrm{K}^{-1}$ the thermal conductivity of nitrogen and $\Omega_{d i s}$ the volume of dissipation, in one bubble.

The attenuation length is larger than the wave extension, so we can use the properties of the inviscid wave propagation to determine the scaling law of the energy dissipation [32].

We first consider the isentropic limit. In that case, the temperature in a bubble during the overpressure is $T+\delta T^{s}$ and varies close to the liquid phase at $T$ over the typical distance $\ell_{T}=\sqrt{\chi^{\tau}}<R$, so that $\Omega_{d i s} \sim R^{2} \ell_{T}$ and $\nabla T \sim$ $\delta T^{s} / \ell_{T}$. Using $\chi=\kappa /\left(\rho_{g} c_{p}\right)$ we get:

$$
\frac{1}{\ell_{a}^{s}} \sim \frac{\dot{E}}{c E_{0}} \sim \frac{c \rho_{0}}{\rho_{g} c_{p} T R} \sqrt{\frac{\kappa}{\rho_{g} c_{p} \tau}},
$$

giving an attenuation length $\ell_{a}^{s} \approx 10 \mathrm{~cm}$ for $\tau \approx 10^{-3} \mathrm{~s}$ and $R \approx 10^{-4} \mathrm{~m}$.

The same kind of analysis can be performed for the isothermal wave propagation. In that case, the gas remains at constant temperature and thus at constant energy (ideal gas assumption). The mechanical work on a 
bubble $\delta W \sim p \delta V \sim R^{3} \delta p$ thus equals the heat exchange with the liquid phase and $\delta Q \sim R^{3} \delta p$ for each bubble, during the characteristic time $\tau$. This heat flux is ensured by thermal conduction and thus $\delta Q \sim \kappa \nabla T^{t} R^{2} \tau \sim \kappa \delta T^{t} R \tau$. From these two relations, we deduce the small temperature variation $\delta T^{t}$ between the bubble center and the bubble boundary. The temperature gradients scale as $\delta T^{t} / R$ and $\Omega_{\text {dis }}=R^{3}$. We finally get:

$$
\frac{1}{\ell_{a}^{t}} \sim \frac{\dot{E}}{c E_{0}} \sim \frac{R^{2} c \rho_{0}}{\kappa T \tau^{2}},
$$

giving $\ell_{a}^{t} \approx 10 \mathrm{~cm}$, like $\ell_{a}^{s}$, for the same parameters.

The dissipation in the continuous phase may also be relevant in the problem. When the foam is compressed, the liquid phase remains incompressible, but the films and the meniscus are sheared or elongated in a complex way which depends on the local foam structure. This induces viscous dissipation in the liquid phase, as well as a specific interfacial dissipation related to the interfacial shear and dilatational viscosities. When the foam is simply sheared, the gas is not compressed and the thermal dissipation is thus probably irrelevant. In contrast, the film and meniscus deformations are a priori of similar nature as under foam compression. Even if the local processes associated to the global deformation of the foam are mostly unknown, the dissipation in the continuous phase can thus be qualitatively estimated from the effective viscosity of the foam measured in simple shear experiments. Kinematic shear viscosities at $100 \mathrm{~Hz}$ of the order of $\nu_{v}=0.02 \mathrm{~m}^{2} / \mathrm{s}$ have been reported [30,31]. The viscous attenuation length $\ell_{a}^{v}$ scales as $1 / \ell_{a}^{v}=\nu_{v} /\left(\tau^{2} c^{3}\right)$ [32], which would lead to $\ell_{a}^{v} \sim 5$ $\mathrm{m}$. As the foam is known to be a shear thinning material, its effective viscosity should be even smaller at higher frequencies. Viscous dissipation is thus a priori negligible in comparison with thermal dissipation.

Whatever their physical origin, all dissipative processes play the mechanical role of an effective viscosity, with $\nu \sim \tau^{2} c^{3} / \ell_{a}$ [32]. From the order of magnitude determined above, $\nu$ is governed by the attenuation (3) for small bubbles and (4) for large ones. The cross-over is reached for $R \sim \ell_{T}$, which corresponds to a maximum of viscosity. This predicts that, in a compressive regime, the effective kinematic viscocity of a foam increases as $R^{2}$ for $R<\ell_{T}$, reaches a maximum and then decreases as $1 / R$, as already proposed in [18].

We define the experimental viscosity by $\nu^{\exp }=$ $b \tau^{2} c^{3} / \ell_{a}^{e x p}$, with $b$ a numerical prefactor close to one. The parameter $b=1.2$ leads to the best agreement with the numerical simulation discussed below. Using this value, we obtained the effective kinematic viscosity $\nu^{\exp }$ for all bubble sizes, as shown in Fig. 4. It lies in the range $[0.2$ - 2] $\mathrm{m}^{2} / \mathrm{s}$ and increases with the bubble radius up to 300 $\mu \mathrm{m}$. These experimental data are fitted by the expression $\nu^{t}=0.4 \tau^{2} c^{3} / \ell_{a}^{t}$, which shows a good agreement for the bubble radii in the range [100-300] $\mu \mathrm{m}$. In contrast, the last point at $500 \mu \mathrm{m}$ is incompatible with the quadratic increase of the viscosity with the bubble size. This radius is much larger than the thermal layer thickness $\ell_{T}=100 \mu \mathrm{m}$, hence the adiabatic limit is reached. The effective viscosity $\nu^{s}=7 \tau^{2} c^{3} / \ell_{a}^{s}$ is plotted in Fig. 4 and reconciles the theoretical and experimental results. Unfortunately, we have not been able to produce foams with larger bubbles and still a $5 \%$ liquid fraction to evidence the viscosity decrease at large bubbles. We have performed experiments at larger $R$, albeit at smaler $\phi_{l}$, that suggest this decrease of attenuation.

Beyond the linear regime, the foam properties must be compared to numerical simulations. A minimal model consists in considering the foam as a compressible and dissipative continuum of density $\rho_{0}$, undergoing weakly nonlinear sound propagation. Hence, we performed numerical simulations based on the second-order equation of sound propagation,

The wave propagation equation is obtained from the series expansion of the compressible, axisymmetric, NavierStokes equation at the second order in the overpressure $p(r, t)=p^{\text {tot }}-p_{0}$ and in the radial foam velocity $u(r, t)$ [33] (eq. 14.4.12):

$$
\begin{aligned}
& \left(\partial_{r r}+\frac{1}{r} \partial_{r}\right)\left(p+\frac{\nu}{c^{2}} \partial_{t} p\right)-\frac{1}{c^{2}} \partial_{t t} p= \\
& -\rho_{0}\left(\partial_{r r}+\frac{2}{r} \partial_{r}\right)\left(u^{2}\right)+\frac{\Gamma}{\rho_{0} c^{4}} \partial_{t t}\left(p^{2}\right),
\end{aligned}
$$

where $\partial_{X}$ and $\partial_{X X}$ respectively stand for partial derivatives of first and second order with respect to variable $X$. The parameter $\Gamma$ is $\rho_{0} c^{4}\left(\partial^{2} \rho / \partial p^{2}\right)_{0} / 2$. For an ideal gas, $\Gamma=(1-\gamma) / 2<0$. The parameter $\nu$ is a dissipative parameter with the dimension of a kinematic viscosity. We only kept first order terms in $\nu$, the validity domain thus being limited by the conditions $p \ll p^{\text {tot }}$ and $\nu \ll c^{2} \tau$ with $\tau \approx 1 \mathrm{~ms}$ the typical duration of pressure variation. This last condition yields $\nu \ll 3 \mathrm{~m}^{2} \cdot \mathrm{s}^{-1}$, with $c \approx 50 \mathrm{~m} / \mathrm{s}$. Practically, reliable results have been obtained for $\nu<2$ $\mathrm{m}^{2} / \mathrm{s}$.

The mass conservation closes the problem and only needs to be computed at order one:

$$
\frac{1}{c^{2}} \partial_{t} p+\rho_{0}\left(\partial_{r}+\frac{1}{r}\right) u=0
$$

We used the phenomenological fit of the pressure $p_{1}(t)$ at sensor 1 shown in Fig. 2 as a boundary condition at the inner boundary. The foam is assumed to be at rest at large $r$. This set of equations is solved numerically with an implicit scheme implemented in a Matlab code, which has been validated with a precision of $1 \%$ using known nonlinear analytical solutions (see Appendix B).

Qualitatively, the viscous term in (5) induces a signal attenuation and a signal spreading, thus leading to an enhanced pressure attenuation. In contrast, the non-linear term acts against the signal spreading, and thus slows down the maximal value decrease. These features, observed experimentally, are reproduced by the model (see 
Fig. 2). However, for large bubbles and large amplitudes, the model fails to predict the high attenuation experimentally observed. Moreover, the front pressure gradient is always underestimated. This could come from the fact that the numerical simulation disregards any acoustic dispersion [21].

The numerical value of the attenuation $\alpha^{\text {num }}$ is plotted in Fig. 3. For each bubble size the dissipative coefficient has been chosen to get the best agreement with $\alpha^{f i t}$. Its value $\nu^{\text {num }}(R)$ is plotted in Fig. 4 as a function of the bubble size. The error bars have been obtained by fitting numerically $\alpha^{\text {fit }} \pm \sigma$.

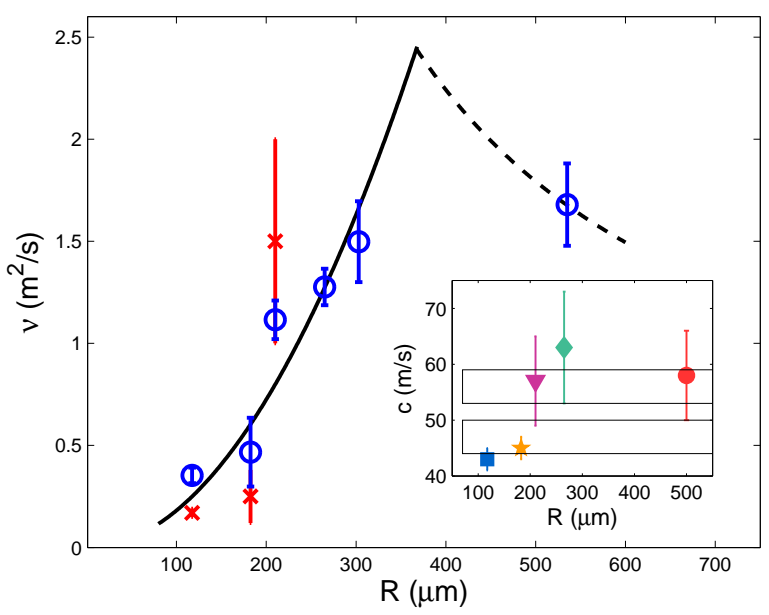

Fig. 4: Foam effective viscosity as a function of the bubble size; (o) viscosities deduced directly from the experimental attenuation, as defined in the text, $(\mathrm{x})$ viscosities obtained as fitting parameter in the numerical simulations, when possible. The error bars are deduced from the experimental dispersion $\sigma(R)$. The solid and dashed lines are the predictions of eq. (4) and eq. (3), rescaled vertically by a factor 0.4 and 7 , respectivelly. Inset: Sound velocity as a function of the bubble size, in the small amplitude limit. Rectangles represent the isothermal (bottom) and isentropic (top) Wood velocities, with the error bar arising from the liquid fraction error bar.

In conclusion, using a foam with a well controlled chemical composition, bubble size and liquid fraction, we show that the main dissipative processes that mitigates a compressive wave, in a time scale domain of the order of $\tau=1$ $\mathrm{ms}$, are of thermal origin. The associated effective viscosity increases as $R^{2} / \tau^{2}$ at small bubbles and departs from this law for larger bubbles. Our theoretical analysis is based on the Wood model. This has been validated by checking that the propagation velocity does not exceed the Wood velocity. It has recently been shown that above a certain radius, Wood's model breaks down and a resonant behavior is expected. For our characteristic time of $1 \mathrm{~ms}$, the resonant bubble radius is of the order of $1 \mathrm{~mm}$ [21]. However strong variations of the sound velocity are expected at the resonance, with a sound velocity of order $200 \mathrm{~m} / \mathrm{s}$ for $R>R_{r}$, which is not compatible with our

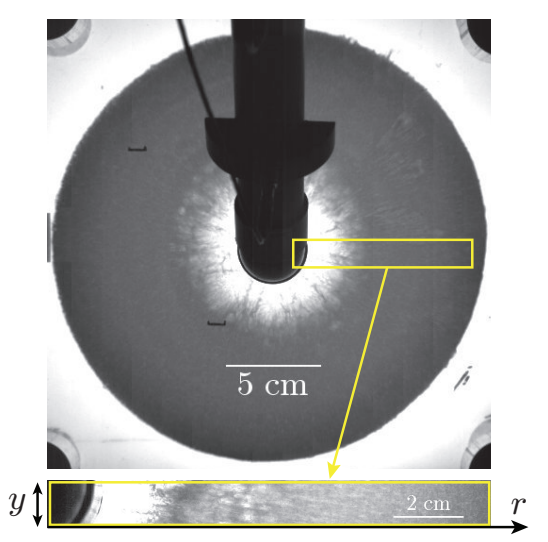

Fig. 5: Image of the foam few ms after the shock. The rectangle is the reduced field of view used for the measurements.

observations. We thus believe that the wave propagation we observe is below the resonance for our whole parameter range (i.e. at a radius smaller than $R_{r}$ ). Moreover, the local process responsible for the attenuation during the resonance was not identified, and the thermal dissipation may be the relevant one.

These results seem limited to compressive waves. However, it is known that plastic events triggered in foams by simple shear induce pressure variations, and thus temperature variations. In this context, the associated thermal dissipation may thus also be important in some parameter range.

$$
* * *
$$

We thank G. Jourdan and L. Houas for their advice on shock tubes and S. Kerampran, C. Clanet, V. Leroy and all the members of ANR SAMOUSSE for enlightening discussions. M. M. acknowledges a financial support by the DGA.

Appendix A. Wave velocity measurements. The foam sample is lightened from below with an intense white light. The foam dynamics is then recorded from top using a high speed camera Photron FastCam Sa3. The camera is triggered by the same signal as the pressure sensor and both measures are therefore synchronised.

Images are recorded with the field of view shown on Fig. 5 (bottom). Each image is averaged in the $y$ direction, and the resulting line is used to produce a space-time plot as shown on Fig. 6. At each position $r$, the first gray level variation is automatically detected by image processing. This first foam motion occurs at time $t^{\mathrm{fr}}(r)$ and coincides with the wave arrival at position $r$. This provides the front wave position $r^{\mathrm{fr}}(t)$.

The wave velocity is deduced from the slope obtained in the linear regime, far from the center.

Appendix B. Code validation. - The set of equations (5) and (6) is solved numerically with an implicit scheme implemented in a Matlab code. Two known ana- 


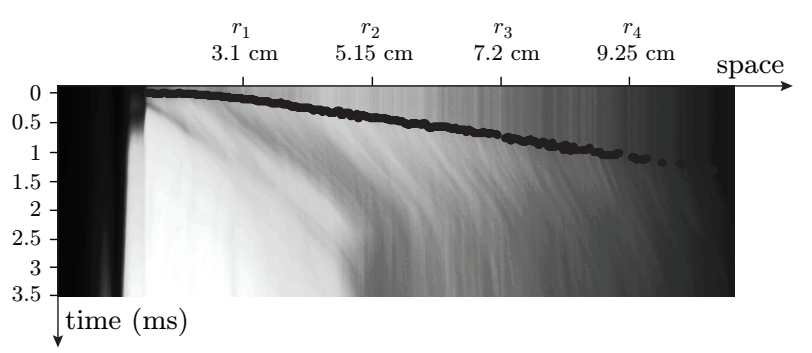

Fig. 6: Space-time image of the foam. The position of the wave front deduced from the image is plotted in black solid circles.

lytical cases have been used to test the code. First, the nonlinear steady solution in a plane wave geometry,

$$
p=p_{0}+\delta p\{1+\tanh [(x-v t) / \delta] / 2\},
$$

with $\quad v=c\left[1+\delta p(1-\Gamma) /\left(2 \rho_{0} c^{2}\right)\right] \quad$ and $\delta=2 \nu \rho_{0} c /(\delta p(1-\Gamma))$. Numerically, setting the inner boundary at the position $r_{0}=3000 \delta$ to be in a plane wave geometry and using the eq. (7) as pressure condition at this point, we got an agreement between numerical and analytical solution better than $1 \%$ on the velocity and on the thickness of the pressure step, for the same time and space discretisation and the same global time and space scales as used in the next section. Second, we tested the energy conservation for an inviscid case at very small amplitude. Using the low amplitude experimental data as boundary condition at the inner boundary (at its experimental position) we found that $\int_{r=0}^{\infty} r p^{2} d r$, which is proportionnal to the wave energy, varies by less than $1 \%$ between $t_{1}^{\max }$ and $t_{4}^{\max }$, for a vanishing viscosity.

\section{REFERENCES}

[1] I. Cantat et al., Foams. Structure and Dynamics (Oxford University Press, Oxford, 2013).

[2] D. Buzza, C.-Y. Lu, and M. E. Cates, J. Phys. II (France) 5, 37 (1995).

[3] A. D. Gopal and D. J. Durian, Phys. Rev. Lett. 91, 188303 (2003).

[4] S. Cohen-Addad, R. Höhler, and Y. Khidas, Phys. Rev. Lett. 93, 028302 (2004).

[5] N. D. Denkov, S. Tcholakova, K. Golemanov, K. P. Ananthapadmanabhan, and A. Lips, Phys. Rev. Lett. 100, 138301 (2008).

[6] T. G. Mason, J. Bibette, and D. A. Weitz, Phys. Rev. Lett. 75, 2051 (1995).

[7] A. J. Liu, S. Ramaswamy, T. G. Mason, H. Gang, and D. A. Weitz, Phys. Rev. Lett. 76, 3017 (1996).

[8] B. P. Tighe, Phys. Rev. Lett. 107, 158303 (2011).

[9] F. Wintzenrieth, S. Cohen-Addad, M. Le Merrer, and R. Höhler, Phys. Rev. E 89, 012308 (2014).

[10] S. Kosgodagan Acharige, F. Elias, and C. Derec, Soft Matter 10, 8341 (2014).

[11] N. Bremond and E. Villermaux, J. Fluid Mech. 524, 121 (2005).
[12] E. Del Prete, A. Chinnayya, L. Domergue, A. Hadjadj, and J.-F. Haas, Shock Waves 23, 39 (2013).

[13] A. Britan et al., Shock Waves 23, 5 (2013).

[14] A. Britan, G. Ben-Dor, H. Shapiro, M. Liverts, and I. Shreiber, Colloids Surf. A 309, 5 (2007).

[15] I. Goldfarb, Z. Orenbakh, I. Shreiber, and F. Vafina, Shock Waves 7, 7788 (1997).

[16] A. Chinnayya, E. Daniel, and R. Saurel, J. Comput. Phys. 196, 490 (2004).

[17] Z. M. Orenbakh and G. A. Shushkov, Acoust. Phys. 39, 63 (1993).

[18] N. Mujica and S. Fauve, Phys. Rev. E 66, 021404 (2002).

[19] I. Ben Salem et al., Soft Matter 9, 1194 (2013).

[20] J. Pierre, R.-M. Guillermic, F. Elias, W. Drenckhan, and V. Leroy, Eur. Phys. J. E 36 (2013).

[21] J. Pierre, B. Dollet, and V. Leroy, Phys. Rev. Lett. 112, 148307 (2014).

[22] J. Pierre, B. Giraudet, P. Chasle, B. Dollet, and A. SaintJalmes, Phys. Rev. E 91, 042311 (2015).

[23] A. Prosperetti, J. Acoust. Soc. Am. 61, 17 (1977).

[24] A. Prosperetti, J. Fluid Mech. 222, 587 (1991).

[25] J. Pierre, F. Elias, and V. Leroy, Ultrasonics 53, 622 (2013).

[26] A. Saint-Jalmes, M. U. Vera, and D. J. Durian, Eur. Phys. J. B 12, 67 (1999).

[27] V. Rodriguez, R. Saurel, G. Jourdan, and L. Houas, Phys. Rev. E 88, 063011 (2013).

[28] A. B. Wood, A Textbook of Sound (MacMillan, New-York, 1930).

[29] V. Surov, High Temperature 38, 97 (2000).

[30] A. D. Gopal and D. J. Durian, J. Colloid Interface Sci. 213, 169 (1999).

[31] K. Krishan, A. Helal, R. Höhler, and S. Cohen-Addad, Phys. Rev. E 82, 011405 (2010).

[32] L. Landau and E. Lifchitz, Physique théorique : mécanique des fluides (Mir, Moscou, 1989).

[33] P. M. Morse and K. U. Ingard, Theoretical Acoustics (McGraw- Hill, New-York, 1968). 